\title{
Impacto del egresado de Terapia Ocupacional de la Universidad Metropolitana. Barranquilla, 2000
}

Fanny Buitrago T. T.O.*, Maitte Flórez L. T.O.**

"Ser terapeuta ocupacional es orgullo de pocos, beneficios de muchos".

\section{PRESENTACIÓN}

Este estudio fue realizado para la celebración del día del Terapeuta Ocupacional en la Universidad Metropolitana, el día 10 de noviembre del año 2000.

\section{OBJETIVO}

Determinar el impacto que ha generado, a través del desempeño laboral, el egresado de la I y II promoción de Terapia Ocupacional de la Universidad Metropolitana en la comunidad Barranquillera.

\section{SÍNTESIS}

El Programa de Terapia Ocupacional de la Universidad Metropolitana, rigiéndose a los parámetros de la Ley 30 de 1992, por la cual se organiza el servicio público de la Educación Superior en Colombia, y en donde se establece el sistema nacional

\footnotetext{
* Terapeuta Ocupacional, Universidad Manuela Beltrán. Diplomada en Gestión Investigativa. Docente Universidad Metropolitana

** Terapeuta Ocupacional, Universidad Manuela Beltrán. Especialista en Docencia Universitaria. Docente Universidad Metropolitana.
} 
de acreditación cuyo proceso tiene como fin fortalecer la calidad de la educación superior preservando así derechos legítimos que tienen los usuarios del sistema y la sociedad global, promueve la necesidad de conocer la situación de desempeño de sus egresados como factor de responsabilidad por reconocer y afrontar las consecuencias que se derivan de sus acciones con relación a las necesidades del contexto en que se opera.

\section{ABSTRACT}

The Program of Occupational Therapy of the Metropolitan University, continuing the parameters of the Law 30 of 1992; which it determine the approaches that govern the Superior Education in Colombia, and where the national system of accreditation settles down whose I process have like end strengthen the quality of the superior education preserving legitimate rights that have the users of the system and the global society, so it promote the necessity of knowing the situation of acting of their graduate like factor of responsibility by recognizing and confront the consequences that are derived of their actions with relationship to the necessities of the context in which it are operated.

\section{METODO}

El presente estudio se enmarca dentro de los lineamientos de la característica 58 del sistema nacional de acreditación, en donde se evalúa el seguimiento de la ubicación y de las actividades que desarrollan los egresados y la preocupación por verificar si esạs actividades corresponden a los fines de la Institución, al compromiso social y al tipo de formación que ofrece; $\mathrm{Al}$ igual que las acciones que viene realizando la Asociación Colombiana de Terapeutas Ocupacionales ACTO, con el fin de fortalecer los requisitos exigidos del ICFES para la creación y mantenimiento de los programas de Terapia Ocupacional a nivel nacional.

El método empleado fue de tipo inductivo-cuantitativo, estableciendo 2 fases o componentes principales:

1. La valoración del profesional en Terapia Ocupacional.

2. La valoración Institucional. 
Esta presentación corresponde a la $1^{\mathrm{a}}$ fase. Mostrando resultados parciales del estudio, la segunda se continuará trabajando, para contar con un registro más preciso sobre el impacto del ejercicio profesional en los diferentes sectores de trabajo y áreas de desempeño.

El número de egresados del Programa de Terapia Ocupacional es de 19, siendo 2 promociones durante el año 2000 específicamente distribuidas en enero y julio. Sin embargo el total de la muestra fue de 17.

Para la realización de este estudio se elaboró un instrumento donde se contemplan los aspectos relacionados a características de la población, sectores de trabajo, áreas de desempeño, cobertura, situación laboràl y proyecciones.

\section{RESULTADOS}

Los resultados que aquí se describen no se dan sobre un $100 \%$ en algunos aspectos, debido a que hay aspectos donde una terapeuta abarca por ejemplo abarca dos sectores de trabajo.

Con relación a la muestra se encuentran desempeñándose profesionalmente el $70.5 \%$, mientras que el $29.4 \%$ al momento del estudio no tenía ningún tipo de vinculación laboral.

El área geográfica de desempeño de las Terapeutas Ocupacionales está distribuido de la siguiente manera:

En un $83.3 \%$ están en Barranquilla, un $16.6 \%$ en Santa Marta y un $8.3 \%$ en Albania (Guajira), Sincelejo y Sabanalarga (Atlántico); con relación a la pròcedencia de estos profesionales al ingreso a la Universidad se registraba que el $58.3 \%$ era de Barranquilla, el $16.6 \%$ de Santa Marta y un porcentaje igual provenían de municipios y el $8.3 \%$ de Sincelejo. Esto nos indica que en relación a la procedéncia y la ubicación laboral los Terapeutas Ocupacionales se encuentran concentrados en Barranquilla, aunque se resalta que se lléga a cubrir regiones como la Guajira y otros municipios cercanos; lo que implica un pobre cubrimiento de la régión atlántica por el poco despliegue de los egresados teniendo en cuenta que el programa es única en esta región. (Ver gráfico No. 1). 


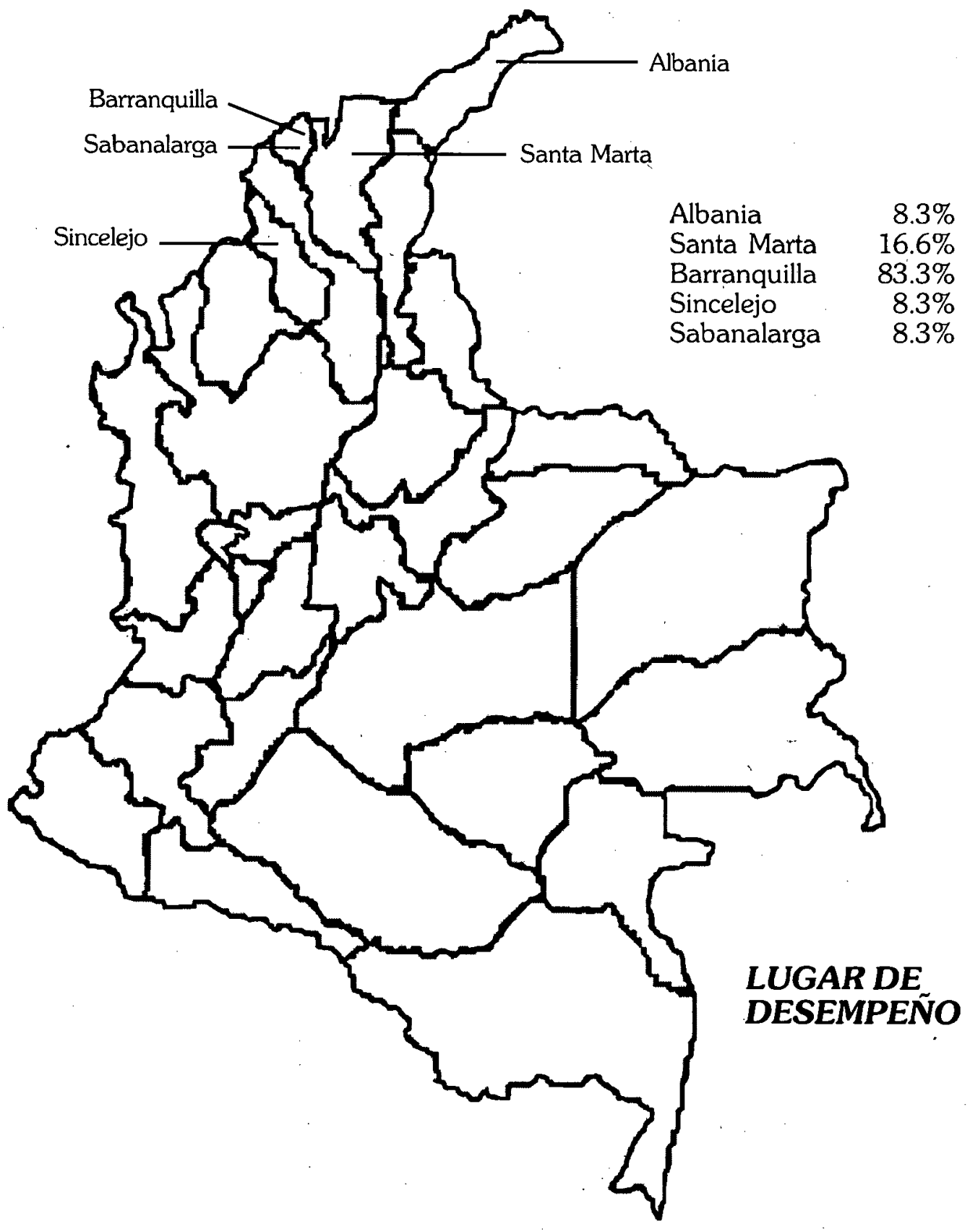


El ingreso al mundo laboral de estos egresados arrojan resultados muy alicientes ya que el $75 \%$ se vinculo en menos de un mes desde su graduación y el $25 \%$ restante en un período de más de dos meses y no mayor a 4 . Si se tiene en cuenta el alto porcentaje en Barranquilla esta vinculación rápida puede deberse a la proyección que como programa a través de su área de extensión ha generado en la comunidad.

En cuanto al Sector Desempeño en el cual se ubican estos egresados se encuentra que:

91.6\% Se concentra en el Sector Clínico

$41.6 \%$ Educativo

$16.6 \%$ Docente

$0 \%$ Laboral

$0 \%$ Justicia Social

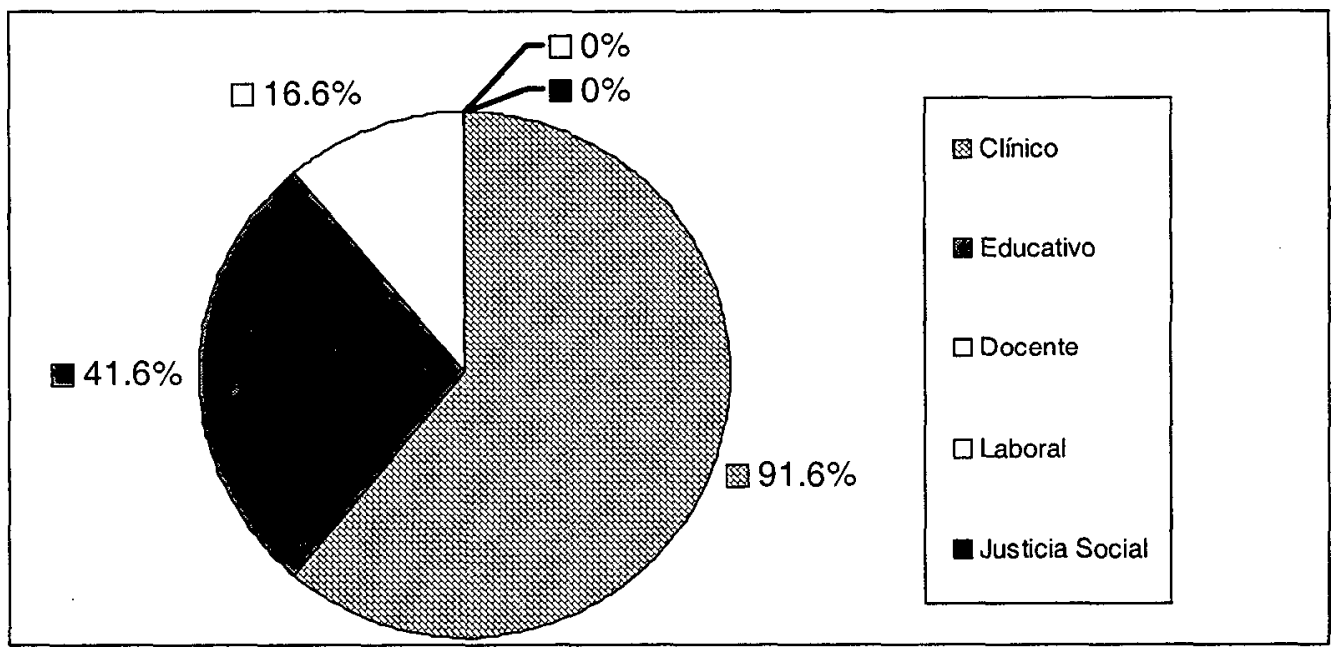

Dentro del Sector Clínico se cubre a través de $8.3 \%$ consultorio particular, $58.3 \%$ Centro de Rehabilitación, $33.3 \%$ clínica, y otro representado en atención domiciliaria un $8.3 \%$.

El sector educativo se cubre a través de: $25 \%$ Colegio Privado, $16.6 \%$ Colegio Público y Educación Especial en igual proporción. 
Lo anterior indica que predomina el sector clínico como área de trabajo y en segundo lugar el Educativo, se anula por completo el área de Justicia Social y laboral. Al igual predomina el Sector Privado y una deficiente participación del Terapeuta Ocupacional a nivel público.

Este resultado aunque en diferente proporción por la muestra se iguala a estudios realizados a nivel nacional en el año 1997 por la Terapeuta Ocupacional Ana María Gómez, en el cual el área de desempeño con mayor acogida es el clínico, en las áreas de disfunción física y psicosocial, siguiendo en orden descendente los sectores de educación, trabajo y justicia social.

Estos resultados pueden basarse en que la creación del Programa se sustento dentro de un contexto clínico para cubrir necesidades a este nivel, pero se resalta que durante el proceso de formación el estudiante aborda todos los sectores de manera teórico-práctico en igual proporción, lo que no implica un énfasis clínico de la Universidad. Otra explicación se da por que donde más se conoce el rol del Terapeuta Ocupacional es en Instituciones de salud, por la demanda de usuarios, lo que tal vez facilita el ingreso de estos profesionales.

Lo anterior nos indica que siendo el sector clínico el que predomina como ubicación laboral, la disfunción física es la más atendida y se cubre especialmente la población infantil por un $91.6 \%$ de los encuestados siguiendo el $66.6 \%$ a la población de adulto y un $25 \%$ adulto mayor; el área de disfunción psicosocial es abordada en un $75 \%$ distribuyendo su atención en un $41.6 \%$ la población Infantil; adulto un $33.3 \%$, y adulto mayor un $25 \%$.

La interacción con otros profesionales corrobora la ubicación del profesional metropolitano en el sector clínico, ya que predominan dentro de los grupos interdisciplinarios médicos (psiquiatra, geriatra), Fisioterapeutas, Fonoaudiologas, Psicólogas, enfermeras y otros. En el Sector Educativo: educador especial, licenciado en Educación, psicopedagogo y en bajísima proporción: administradores, ingenieros y técnicos en Salud Ocupacional.

Con relación a la situación actual del egresado está enmarcado dentro del régimen de la Ley 100 de Seguridad Social y la Legislación Laboral dentro del código sustantivo del trabajo, encontrándose que: 
- La distribución de la jornada laboral es de:

$58.3 \%$ cubre una jornada de tiempo completo

$33.3 \%$ medio tiempo

$33.3 \%$ porhoras

- El número de sitios de trabajo es: el $41.6 \%$ labora en 2 sitios de trabajo el $1.6 .6 \%$ en 3 sitios

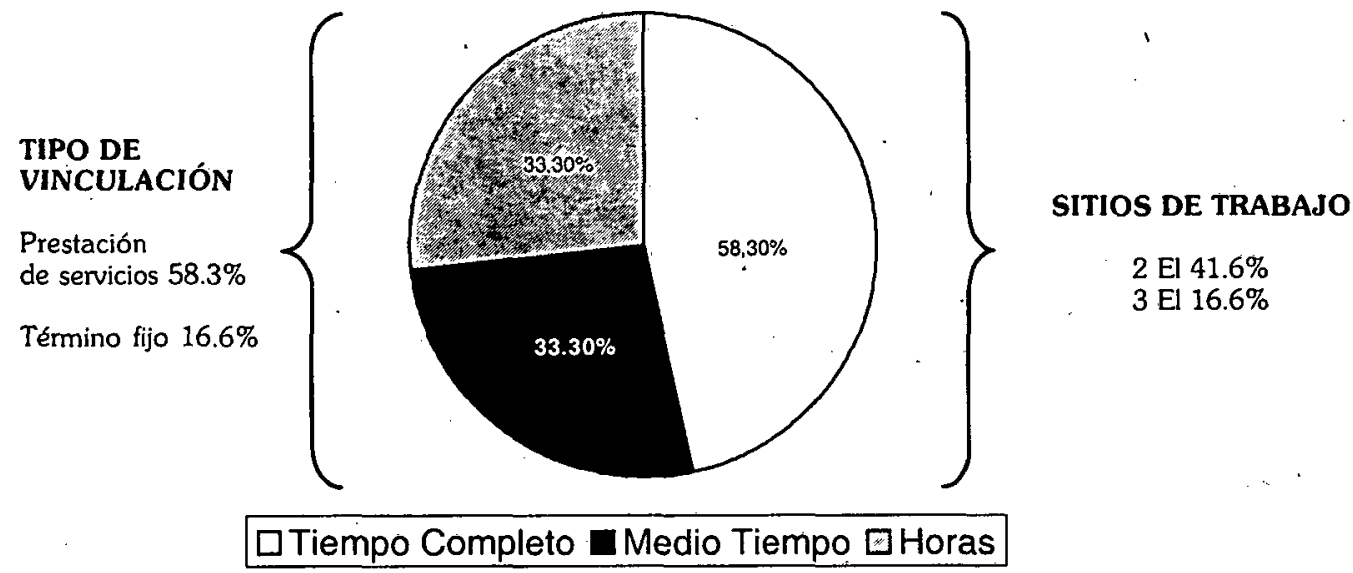

- El tipo de contratación esta dada por:

Prestación de Servicios en un 58.3\%, y Contratación Término Fijo de un 16.6\%

- El Promedio de ingreso mensual está dado entre:

$25 \% \rightarrow 3-4$ salarios mínimos (SM)

$25 \% \rightarrow$ No responde

$16.6 \% \rightarrow 2-3 \mathrm{SM}$

- La vinculación a estas Instituciones se dio por:

* Que existía una vacante en la Institución: $83.3 \%$

* Venta del servicio: $16.6 \%$

* Creación de programas y propuestas en el $50 \%$

Los resultados anteriores indican baja gestión empresarial (Paralelo a la situación económica del país, y a la poca experiencia profésional) ya que, la visión esta dada 
a pedir empleo, lo que se facilita cuando ya han sido creados los servicios o cargos en las Instituciones y se encuentran vacantes. Sin embargo esta situación afecta a los sectores que desconocen el papel del terapeuta ocupacional, fenómeno que se corrobora en los resultados expuestos anteriormente, debido, tal vez, a la pobre creación de programas o propuestas por parte de los egresados metropolitanos.

Dentro de la dedicación de funciones en su ejercicio profesional, se encuentra que para el

Area Asistencial $\quad \rightarrow 34$ Horas semanales.

Area Administrativa $\rightarrow 12$ Horas Semanales.

Area Docente $\rightarrow 11$ Horas Semanales.

Area Investigativa $\rightarrow$ 0 Horas semanales.

Lo anterior permite entrever un quehacer repetitivo, de aplicación de conceptos en donde las tareas administrativas e investigativas que tienen poca o nula representación no muestran un desarrollo lo que puede debilitar el quehacer profesional y el impacto en la región.

Teniendo en cuenta que como profesión existe ciertos saberes básicos sin los cuales no es posible responder a las expectativas sociales con calidad o responsabilidad. Estos saberes incluyen conocimientos, habilidades y técnicas indispensables para el ejercicio profesional, incluye además una ética ligada a los efectos sociales de la acción y un conjunto de normas implícitas y explícitas que se espera guíen el comportamiento general del profésional, se indagó sobre la percepción que se tiene de la congruencia o no de la formación profesional con respecto a la realidad laboral encontrándose que el:

- $83.3 \%$ considera que se dio lo fundamental a través de la teoría y la práctica, pero es deber de cada uno profundizar.

- $8 \%$ considera que faltaron conocimientos.

Esto se relaciona con la percepción que se tiene del impacto en el área de trabajo, ya que el $100 \%$ considera que el Terapeuta Ocupacional Metropolitano ha generado por sus conocimientos, por los resultados obtenidos, por las preguntas innovadoras un impacto positivo en su contexto.

Dentro de las proyecciones a mediano y largo plazo de los egresados Metropolitanos, se encuentra: 
- Búsqueda de Profundización o experiencia.

- Especialización (docencia, Salud Mental, Salud Ocupacional. 1\% en el exterior).

- Mejor ubicación laboral.

- Independizarse.

\section{CONCLUSIONES}

Esta fase del estudio permite concluir a las investigadoras que:

1. El impacto generado se ha centrado en la ciudad Barranquilla, observándose regiones desprotegidas, creándose las expectativas en que futuros profesionales cubran estas áreas.

2. Se observa que el Terapeuta Ocupacional Metropolitano formado bajo una visión dialógica e interaccional ha liderado procesos en sus sitios de trabajo gestando proyectos aunque es clara la ausencia de acciones en proyecciones a nivel gubernamental $u$ Organizaciones no Gubernamentales que cubren los sectores no intervenidos actualmente, laboral-justicia social.

No se percibe agremiación a nivel nacional o internacional, ni se tiene en cuenta en las proyecciones profesionales.

El Promedio de ubicación laboral es alentador lo que no es compensado en tiempo, remuneración y tipo de vinculación laboral teniendo en cuenta claro esta situación social y legislativa del país y la poca experiencia en tiempo del egresado, lo que debe motivar a los profesionales y estudiantes a gestionar en otros sectores en donde puede existir un mayor reconocimiento profesional y económico del Terapeuta Ocupacional.

3. Que el Terapeuta Ocupacional Metropolitano está generando un impacto en el Sector Clínico en las áreas de Función y disfunción física y abordando especialmente la población infantil.

4. Hay una alentadora incursión en el sector educativo. 


\section{RECOMENDACIONES}

1. El presente estudio permite plàntearle al Programa la revisión de su estructura curricular de acuerdo a las demandas actuales en el ámbito político, social y demográfico de la región.

2. Revisión de los aspectos de extensión de manera que se cubran otros sectores en la región y proyectar así el quehacer profesional abriendo espacios laborales.

3. Para los egresados se hace necesario, a través de educación continuada, la capacitación en gestión de proyectos que les permita liderar su propia empresa, teniendo en cuenta que en el marco mundial este aspecto es factor importante de la economía de un país.

4. Igualmente es fundamental la concientización de la importancia de la acción profesional, ya que mediante este aspecto y una adecuada práctica de conocimientos se puede proyectar la Terapia Ocupacional en otros ámbitos.

5. A la población en formación es vital el desarrollo del espíritu investigativo con incentivos para el fomento del conocimiento científico que genere aportes significativos a nuestra disciplina.

6. Se debe continuar este estudio en su II fase para cumplir con el objetivo propuesto. 